\title{
The Inspection of Wind Blades Using Thermography with Different Exciting Methods
}

\author{
Xiao-Li LI ${ }^{1 \mathrm{a}}$, Da-Peng CHEN ${ }^{2}$, Zhi ZENG ${ }^{3}$, Jing-Ling SHEN ${ }^{3}$, Xun WANG ${ }^{3}$ and \\ Yue-Jin $Z \mathrm{HAO}^{1 \mathrm{~b}^{*}}$ \\ ${ }^{1}$ School of Optoelectronics, Beijing Institute of Technology, Beijing, China \\ ${ }^{2}$ Science and Technology on Optical Radiation Lab., Beijing, China \\ ${ }^{3}$ Deptmentof Physics, Capital Normal University, Beijing, China \\ a3120140271@bit.edu.cn, byjzhao@bit.edu.cn
}

Keywords: Wind blade,Thermography, Defect, Exciting methods,

\begin{abstract}
In this paper, different exciting methods of active thermography, including the pulsed flash lamp exciting, halogen lamp step heating and ultrasonic exciting, were applied to detect different kinds of defects according to their different heat build-up properties. The results showed in the paper reveal that the themography is an available means to inspect some common defects in the wind blades. The paper also advises that use the flash lamp exciting for inspecting near surface defects, the halogen lamp step heating method for deeper ones, and ultrasonic exciting method for defects which have adjacent interfaces.
\end{abstract}

\section{Introduction}

Wind power is considered to be a renewable and clean energy, catching more and more attentions all over the world. Wind blade is one of the most important parts for the wind power system, and it is made of composite materials. With the development, the blade is getting larger, longer and more powerful. The longest blade in the world was reported recently to be 83.5 meters. Many kinds of defects such as pin hole, delamination, debonding, hungry joint, air bubbles, impact damage and crack may occur during the procedures of fabricating, transporting, installing and employing. Because the wind blade is of large size and has complicated structures and materials, what is more, it worksin harsh environment, and defects may appear in not only near surface but also very deep location. But unfortunately, most of the domestic companies have to apply the process controlling method in manufacturing and visual or tapping inspection in serving because of that there is no mature NDT(nondestructive testing) methods to solve the inspection problems. If impact damage, wind erosion, crack or other big defects appear, mending steps need to be carried out. However, neither degree of the defects nor the repair quality can be evaluated by a scientific NDT method. In recent years, many testing technics such as thermography, acoustic emission, ultrasonic testing, X-ray inspection and etc. have been developed in the international NDT field [1-4], but all of them are under experimental research. Traditional NDT methods are not so applicable to the field or in-service testing because of their technique limits. Thermography is one of the most promising technologies for the reasons that it is noncontact, single-side access, wide-area and fast, strong field adaptability, applicable to very wide range of materials especially composites, unaffected by the geometry and providing a simple clear image results of the inspection area, and so on. In this study, thermography with three different exciting methods including pulsed flash lamp exciting, halogen lamp step heating and the ultrasonic exciting are applied to inspect defects in real wind blades or samples, and the applicability of those sources will be discussed.

\section{Methods}

Since 1990s, many government organizations and large multinational corporations in developed countries build infrared thermal nondestructive testing laboratories in order to develop thermography 
technology and application research for advanced and engineering materials/structures, so as to solve their unique nondestructive testing issues. During past three decades, thermography has been evolved into many branches which were mainly different in exciting technologies or data progressing methods, such as pulsed thermography, sonic infrared imaging, electromagnetic thermal imaging, lock-in thermography, etc...The theory of thermography is based on heat conduction in solids and thermal radiation of the surface. Fig. 1 is the schematic drawing of the radiation exciting test; it is also an example of how defects and structures can be detected by thermography. The heating source heats the surface of the sample, at the same time the surface temperature raises. While the heat propagates from the surface to the inner of the sample, the surface temperature gets down. The cooling process is determined by the material properties and boundary conditions of the specimen. Normally, the existence of any discontinuities or in homogeneity under the surface, such as defects, damage and structures will change the surface cooling process of the corresponding area. The IR camera is involved to record the whole process of the surface temperature variation including before and after heating. Hopefully, the information of defects, damage and discontinuity can be checked out after the data analyses and processing.
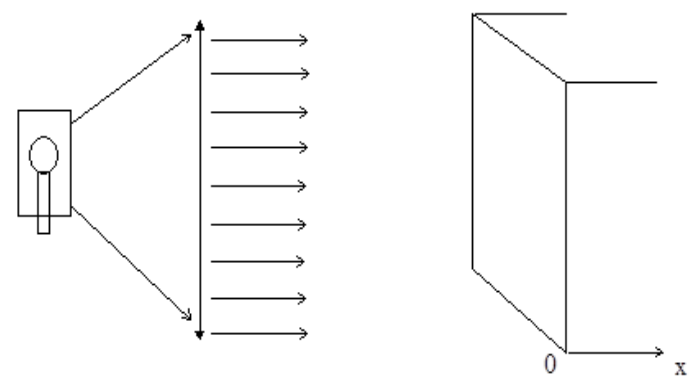

Fig. 1.theSchematic Drawing of the Test

Ignoring the horizontal heat conduction, the general thermal diffusion equation is given by

$$
\nabla \cdot[\mathrm{k} \nabla T(\mathrm{x}, \mathrm{t})]-\rho \mathrm{C}_{\mathrm{v}} \frac{\partial \mathrm{T}(\mathrm{x}, \mathrm{t})}{\partial \mathrm{t}}=-\mathrm{f}(\mathrm{x}, \mathrm{t})(1)
$$

where $\mathrm{k}$ is the thermal conductivity of the sample, $\mathrm{T}(\mathrm{x}, \mathrm{t})$ is the temperature at position xand time $\mathrm{t}, \rho$ and $c_{v}$ represent the density and specific heat separately. $f(\mathrm{x}, \mathrm{t})$ is the heat source function which gives how the heating is applied to a medium.

Unlike the conventional passive infrared testing technic that only monitors the surface temperature variations passively; active thermography employs various heating techniques to actively excite the subsurface defects and flaws. It is vital to know the interactions between controlled heating and subsurface thermal in homogeneity. Furthermore, the selection of an appropriate exciting method is crucial for a successful test. In practical wind turbine application, a comprehensive exciting approach may develop in order to inspect different kinds of defects at different depth.

\section{Pulsed Exciting Thermography}

For a pulsed exciting to the surface, the source function in Eq. 1 can be written as

$$
f(x, t)=q \delta(t) \delta(x)(2)
$$


where q represents the total input energy per unit area.

Thermal diffusion equation in Eq. 1 can be written as

$$
\frac{\partial^{2} T(x, t)}{\partial x^{2}}-\frac{1}{\alpha} \frac{\partial T(x, t)}{\partial t}=-\frac{q}{k} \delta(t) \delta(x)(3)
$$

The solution of Eq. 3 in a semi-infinite isotropic solid is given by [5-6]

$$
\Delta \mathrm{T}(\mathrm{t})=\frac{q}{e \sqrt{\pi t}}(4)
$$

Wheree $=\sqrt{k \rho c}$ is thermal effusivity, and the $\alpha=\frac{k}{\rho c}$ is the thermal diffusivity. When there is subsurface defect or structure difference, the one dimensional solution is given by [5-6]

$$
\Delta \mathrm{T}(\mathrm{t})=\frac{q}{e \sqrt{\pi t}}\left[1+2 \sum_{n=1}^{\infty} \exp \left(\frac{-n^{2} d^{2}}{\alpha t}\right)\right](5)
$$

Where d is defect depth.

\section{Step Heating Thermography}

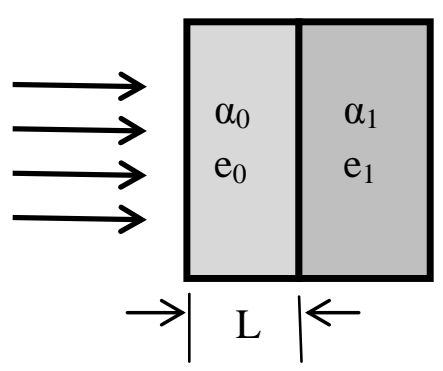

Fig. 2.The Schematic Drawing of the Two-layer Sample Test

As shown in Fig. 2, the surface layer of thickness is $\mathrm{L}$ and its properties $\alpha_{0}, \mathrm{e}_{0}$ separately represent diffusivity and effusivity; a backing layer also has properties of $\alpha_{1}, e_{1}$. The surface temperature for step heating is given as a function of time t. [7]

$$
T_{S}(0, t)=\frac{P_{0}[1-R(\lambda)] \sqrt{t}}{\sqrt{e_{0} \pi}}\left\{1+2 \sum_{n=1}^{\infty}(-\Gamma)^{n}\left[\exp \left(-\frac{n^{2} L^{2}}{\alpha_{0} t}\right)-\frac{n L \sqrt{\pi}}{\sqrt{\alpha_{0} t}} \operatorname{ErFc}\left(\frac{n L}{\sqrt{\alpha_{0} t}}\right)\right]\right\}
$$

where $\Gamma=\mathrm{e}_{1}-e_{0} / \mathrm{e}_{1}+e_{0}(7)$

where $P_{0}$ is the intensity of the light modulated with frequency, $R(\lambda)$ is the wavelength dependent reflectivity at the surface, $\Gamma$ is thermal mismatch factor or thermal reflection coefficient, and $\operatorname{ErFc}(\mathrm{x})=1-\operatorname{ErF}(\mathrm{x})$ where $\operatorname{ErF}(\mathrm{x})$ is the error function. 


\section{Ultrasonic Exciting}

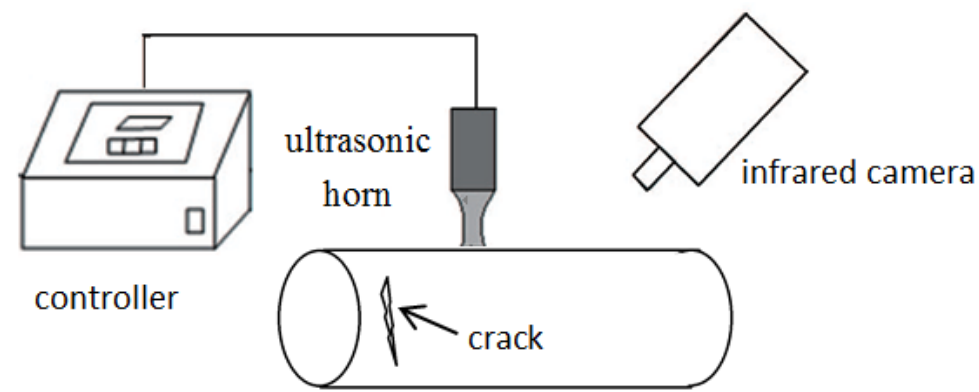

Fig. 3. The Schematic Drawing of the Ultrasonic Thermography

The experiment setup of ultrasonic thermography is shown in Fig.3. The horn transduces the energy of vibration as the form of ultrasonic to the sample; adjacent interfaces, such as crack or debonding, will rub with each other and result in high temperature, so that we can find it with the help of an infrared camera.

After the $n$th impulse, the solution of vibration amplitude is [8]

$$
x_{n}=A_{n} \cos \omega n \tau+B_{n} \sin \omega n \tau
$$

here, the coefficients $A_{n}$ and $B_{n}$ are given by

$$
\mathrm{A}_{\mathrm{n}}=\mathrm{A}_{1}+\frac{\mathrm{A}}{\omega} \sin \pi \mathrm{n}\left(\frac{\omega}{\Omega}\right)\left[\cos \pi \mathrm{n}\left(\frac{\omega}{\Omega}\right)-\cot \pi \mathrm{n}\left(\frac{\omega}{\Omega}\right) \sin \pi \mathrm{n}\left(\frac{\omega}{\Omega}\right)\right]
$$

And

$$
\mathrm{B}_{\mathrm{n}}=\mathrm{B}_{1}+\frac{\mathrm{A}}{\omega}\left\{\sin \pi \mathrm{n}\left(\frac{\omega}{\Omega}\right)\left[\sin \pi \mathrm{n}\left(\frac{\omega}{\Omega}\right)+\cot \pi \mathrm{n}\left(\frac{\omega}{\Omega}\right) \cos \pi \mathrm{n}\left(\frac{\omega}{\Omega}\right)\right]-1\right\}
$$

Where $\omega$ is the natural frequency of the oscillator, $A$ is the impulse strength, $A_{1}$ and $B_{1}$ are the original amplitudes, and $\Omega=(2 \pi / \tau)$ is the angular impulse frequency. This set of equations is periodicif $(\omega / \Omega)$ is a rational fraction, and possibly to be a resonance exists.

\section{Results}

\section{Pulsed Flash Lamp Exciting}

Pulsed thermography is one of the most common techniques in thermography NDT. Flash lamp exciting provides a pulsed heating and often used in quantitative research for the reason that it has relatively simple physical models. This techniqu e is wildly applied in military and civilian aerospace for testing debonding, delamination, impact damage, inclusions, foreign object debris, and water or oil ingress in many composite materials. A lot of research institutes and large companies including Boeing, Lockheed Martin, Pratt and Whitney, Rolls-Royce, NASA, U.S. Army, Navy and Air Force, Ford, General Motors, have introduced pulsed thermography. While in testing wind turbine blades, it usually used for inspection near surface defects, such as g offer, air bubbles, pin holes, etc. because 
of that it supplies relatively low energy. Many pulsed thermography results had reported by Zhi Zeng, Ning Tao, Dahao Yue, etc. [9-11]

There are mainly several factors to consider in designing the wind turbine blades: aerodynamics, strength and stress of the structure, noise and so on, which is mainly involved in geometry, weight and its balance. The wooden and PVC foam core in the wind turbine blades play the role of improving stiffness, increasing stability and reducing the weight of the blade. The gap will affect both the weight and load distribution in the blades. According to the quality requirements, the gap between the core and its surroundings larger than $3 \mathrm{~mm}$ is not allowed. The inspection area shown in Fig. 2 was chosen from a real wind turbine blade. There is about $3 \mathrm{~mm}$ glass fiber reinforced material covering on the cores.

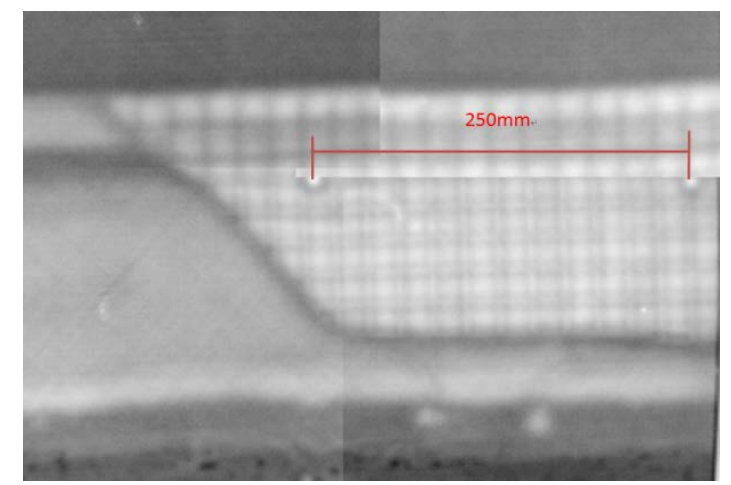

Fig. 2. Thermal Image of the Gap between Wooden Core and Its Surroundings

The inspection area was separated in four pieces before testing because of that the area is beyond the single shoot of inspection area. Excite the four areas separately and joint those as one when analyzing the results. Markscan help us fix the position while jointing the thermograms. The distance between two marks is $250 \mathrm{~mm}$. The excitation flash power employed in this test was 9.6KJ, and the pulse length was $2 \mathrm{~ms}$. The capturing rate of the camera was $60 \mathrm{~Hz}$.

The state of the gap between wooden core material and its surroundings is shown in the Fig. 2 . There are wooden cores, PVC cores, glass fiber reinforced plastic, and little bubbles in the thermogram. Due to that a specific length was given in the infrared image, the size of region of interest can be deduced through the ratio with the given length. That's the way to get the gap distance. The resolution of the thermograpm can be improved by using a higher optic resolution camera in the test. The flash lamp exciting thermography is proven to be a good method to check gap distance. 


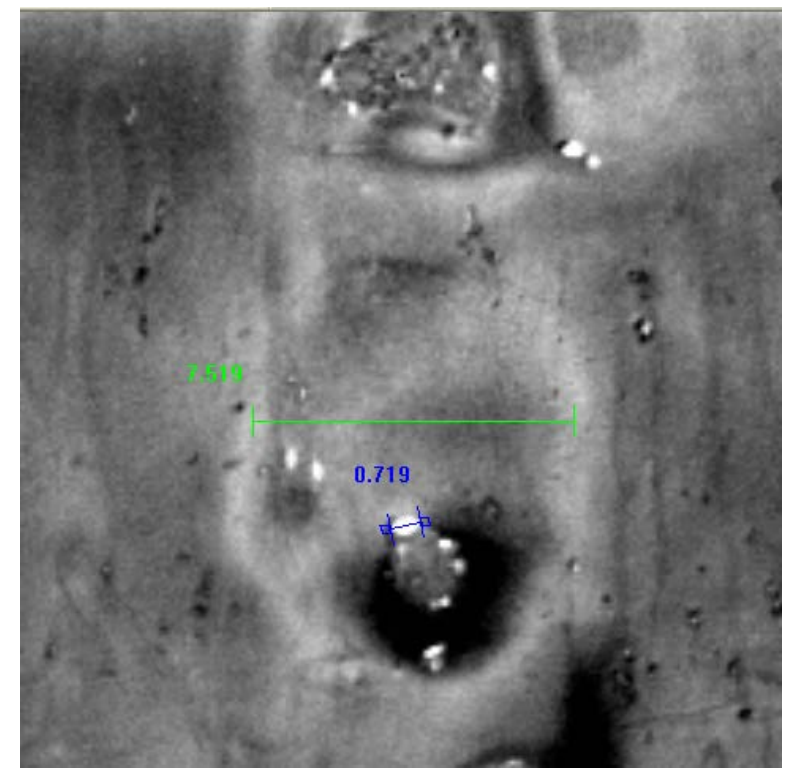

Fig. 3. Thermal Image of Undercure

Very low viscosity epoxy resin impregnating material is largely used in wind turbine blades for its excellent physical and mechanical property. It can meet the requirement of the blades to work no less than 20 years in harsh environment. Cure process including the dose of the curing agent and accelerator, cure temperature, cure time will influence the cure quality. A problem developed in any part of the process may result in undercured defect. Usually, the undercured epoxy resin has the shortcomings of uneven density, internal stress, brittle and bad impact resistance, and so on. As a result, crack may develop at the undercure area in the wind turbine blades and cause great loss. Fig. 3 shows the flash lamp exciting thermography result of under cure. From the thermogram different degree of cure at different areas was displayed. Besides, several small bubbles were also detected.

\section{Halogen Lamp Step Heat}

Because the wind turbine blades has huge shape, for instance, the recent mainstream 1.5MW wind turbine bladeis 42.5 meters long and 6 tons weighs, defects may develop at any depth, not only near surface. As a result, halogen lamp step heat method that can supply more power is needed to inspect deep flaws. Comparing with flash lamp exciting, step heating method can adjust its exciting energy through working time. The following results in this section were excited by two 1000W halogen lamps.

The beam is one of the most important supporting structures in the wind turbine blades. The defects in beam will directly harm the quality and shorten the service life of the wind turbine blades. The delamination is a common defect in the beam. The specimen in Fig. 4 is made of glass fiber, and is used to simulate the delamination defect using plat bottom holes. Its size is about $270 \mathrm{~mm}$ by $240 \mathrm{~mm}$. The plat bottom holes are made in three rows with depth of $2 \mathrm{~mm}$, 3mm and $4 \mathrm{~mm}$ to the surface, and each row has 6 holes with different diameters. 


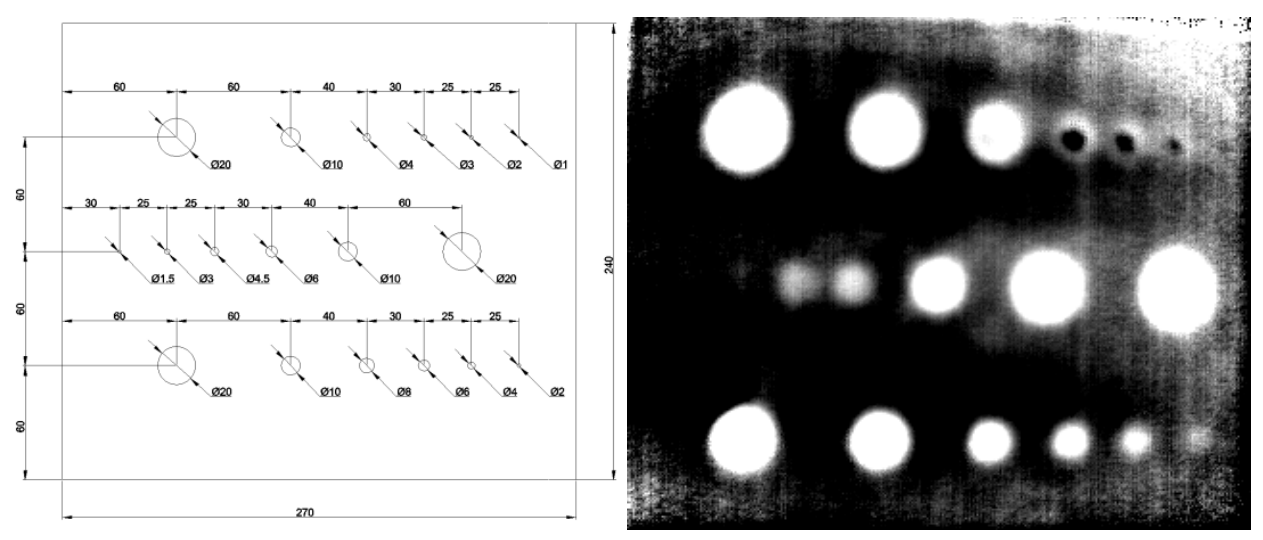

Fig. 4. The Design and Thermogram of Glass FiberPlate

Excite the surface 20secondswith halogen lamps; capture the variation of surface temperature before and after exciting using an infrared camera. After reconstructing the data, we will get the thermogram in Fig. 4.

Basically, the deeper and smaller the defect is, the more difficult to detect. Among the holes in the thermogram in Fig.4, two out of three smallest diameter-to-depth holes was inspected, they were a $2 \mathrm{~mm}$ diameter to $2 \mathrm{~mm}$ deep hole and a $4 \mathrm{~mm}$ diameter to $4 \mathrm{~mm}$ deep hole. To some extent, it represents the detection ability of the testing system to this material.

With the development of increasing capacity and breeze-generated electricity technology, the weight reduction of the wind turbine blades is a key technic. The carbon fiber beam is researched to replace the glass fiber beam in order to reduce weight. The delamination is also one of the most common defects in the carbon fiber beam, just like the glass fiber one. As shown in Fig.5, a specimen whose raw material comes from a section of real carbon fiber beam is designed. Eight plat bottom holes with $20 \mathrm{~mm}$ diameter and $1 \mathrm{~mm}$ to $8 \mathrm{~mm}$ depth are dedicated to simulate the delamination. All of the fibers have the same orientation, so the material is thermal anisotropic. The fiber orientation has relatively fast conductivity than its vertical orientation. That is the reason why the simulated circular holes appeared as elliptical holes in the thermogram. And this thermal anisotropic character affects the testing results. All the simulated defects in this sample can be detected using this technic.

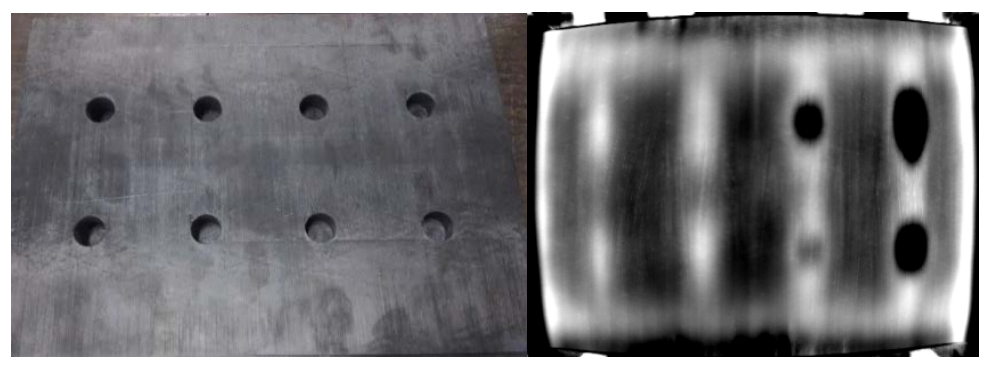

Fig. 5Thermogram of Carbon Fiber Plate Delamination Similation Specimen

The wind turbine blade has complicated structure, consists of two halves and one or two main spars, all of which are joined with glue. Zhi Zeng and Ning Tao showed the flash lamp exciting results of adhesive detection in their papers [5-6].In most cases, the bounding areas between main spar and beam bury deep, which is difficult to inspect using flash lamp exciting method. Fig. 
6shows the thermograms of glass fiber plate adhesive specimens for hungry joint and air bubbles in glue. Both specimens measure $110 \mathrm{~mm}$ square and $4 \mathrm{~mm}$ depth with the glue.
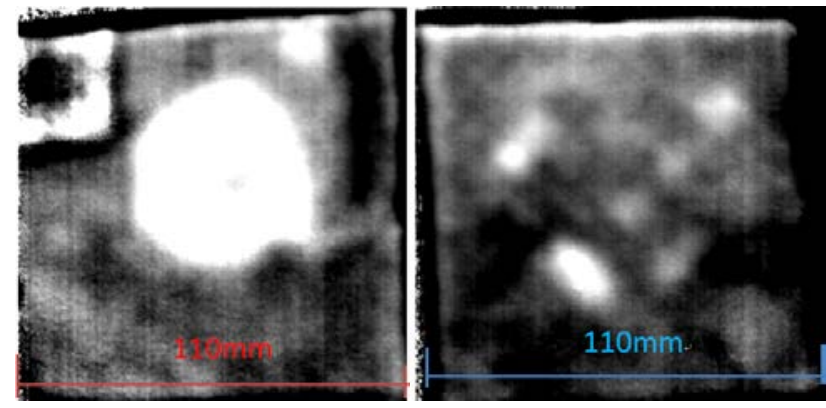

Fig. 6. Thermograms of Glass Fiber Plate Adhensive Specimens for Hungry Joint and Air Bubbles in Glue

Compare with the flash lamp exciting method, halogen lamp step heat is more suitable for inspecting low conductivity or thick materials.

\section{Ultrasonic Exciting}

Vibrothermography is another active thermography NDT technique. The ultrasonic excitation will cause mechanical vibrations to the sample. And the vibration induced external to the structurewill cause rubbing at the surfaces of the defects which may present, resulting in friction and generating heat. This exciting technique can heat the surface or subsurface of the defects. What's more, it will not heat the sound areas. These characters make it possible to find the defects at the first time. X. Han, Z. Zeng, and D. Chen etc. had shown many sonic infrared imaging results in their papers [12-14].The sonic infrared imaging technology is proven to be a powerful technique for detecting defects such as cracks, debonding and delamination. While the traditional ultrasonic gun needs to contact the specimen with a certain pressure, that may cause some damage to the surface. The air-coupled ultrasonic exciting technic was revealed in recent years, but it still under research. [15]

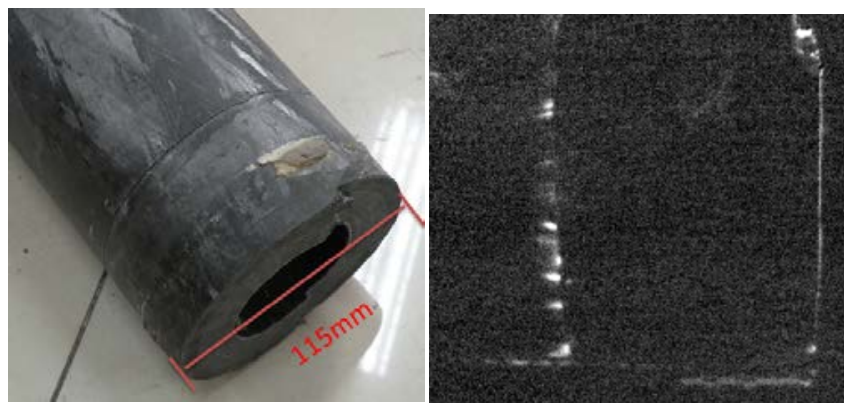

Fig. 7. Picture of Carbon Fiber Tube and the Thermogram of the Crack

A carbon fiber tube shown in Fig. 7 has a diameter of $115 \mathrm{~mm}$, and the wall thickness is $27 \mathrm{~mm}$. There is a big visible crack in it. The tube is made for adjusting direction of the wind turbine blades.

The specimen is excited with anultrasonic gun whose frequency is $20 \mathrm{KHz}$ and electric power is 4000W. Hold the exciting about 5seconds, the temperature at the crack will rise. The thermogram in Fig. 7 shows us a high reservation of the crack. During the vibration, the closer of the two faces the higher the temperature rise, because close interfaces are easily to cause more friction and generate more heat. 


\section{Conclusions}

The paper shows the thermograms of defects in the wind blade such as undercure, delamination, hungry joint, air bubbles and crack. The results reveal that thermography is an effective technology to inspect some kinds of defects in wind blades.

Comparing with these three exciting methods mentioned above, the pulsed flash lamp exciting is more suitable for near surface defects, and the halogen lamp step heat fits for deeper ones better. However, those defects have adjacent interfaces is advised to choose the ultrasonic exciting method.

\section{Acknowledgement}

This work was supported in part by Innovation Ability Promoting Project to City-belonging University of Beijing Education Committee, in part by National Science Foundation of China No.U1233120 and No.61079020.

\section{References}

[1] MA Drewry, GA Georgiou, A Review of NDT Techniques for Wind Turbines,Insight,49(2007)137-140.

[2] Peter Meinlschmidt, Jochen Aderhold, Thermographic Inspection of Rotor Blades, ECNDT, Berlin, 2006.

[3] Anne Juengert, Inspection Techniques for Wind Turbine Blades using Ultrasound and Sound Waves, Non-Destructive Testing in Civil Engineering, Nantes, 2009.

[4] Anne Jüngert, Damage Detection in Wind Turbine Blades using two Different Acoustic Techniques, $7^{\text {th }}$ fib PhD Symposium in Stuttgart, Germany, 2008.

[5] D. P. Almond, S.K. Lau, Defect sizing by transient thermography I. An analytical treatment, J. Phys. D Appl. Phys, 27(5)(1994)1063-1069.

[6] Zhi Zeng, Jing Zhou, Ning Tao, LichunFeng, Cunlin Zhang, Absolute peak slope time based thickness measurement using pulsed thermography, Infrared Physics \& Technology, 55(2012)200-204.

[7] Xavier P.V. Maldague, Patrick O. Moore, nondestructive testing handbook of Infrared and Thermal Testing, third edition, ASNT, 2001.

[8] Xiaoyan Han, Wei Li, Zhi Zeng, Acoustic chaos and sonic infrared imaging, Applied Physics Letters, 81(17)(2002)3188-3190.

[9] Zhi Zeng, Ning Tao, LichunFeng,Relative thermal contrast analysis in the inspection of wind turbine blades using pulsed thermography, Advanced Materials Research, 301-303(2011)591-596.

[10]Ning Tao, Zhi Zeng, Lichun Feng, the application of pulsed thermography in the inspection of wind turbine blades, SPIE, Beijing, 2011.

[11] Yue Dahao, Li Xiaoli, Zhang Haojun, IR Thermography Inspection of Wind Turbine Blades, Infrared Technology, 33(2011)614-617. 
[12] Xiaoyan Han, Jianping Lu, Md. Sarwar Islam, Wei Li, Zhi Zeng, Sonic infrared imaging NDE,SPIE, Bellingham, 2005.

[13]Zhi Zeng, Jing Zhou, Ning Tao, Support vector machines based defect recognition in SonicIR using 2D heat diffusion features, NDT\&E International, 47(2012)116-123.

[14] Xiaoyan Han, V. Loggins, and Zhi Zeng, L.D. Favro, R.L. Thomas, Mechanical Model for the Generation of Acoustic Chaos in Sonic IR Imaging,Applied Physics Letters,85(2004)1332-1334.

[15]Chen Dapeng, Zhi Zeng, Cunlin Zhang, Air-coupled ultrasonic infrared thermography for inspecting impact damages in CFRP composite,Chinese Optics Letters, 10(2012)S10401. 